\section{Playlist-Sammlungen für} die optimale Facharzt-Vorbereitung: die Weiterbildungscurricula in der Thieme eRef

Denken Sie bei dem Begriff „Playlists“ auch sofort an Streamingdienste wie Spotify oder Youtube? Sie begegnen uns mittlerweile fast täglich, sogar bei der Thieme Wissensplattform eRef finden Sie das PlaylistKonzept und es funktioniert ähnlich: ein Titel, viele Quellen, nur die besten Inhalte, strukturiert angeordnet.

Diese praktischen Zusammenstellungen relevanter Inhalte zu ausgewählten Themen sind beispielsweise Kernstück der Weiterbildungscurricula in der eRef, die von jungen Ärztinnen \& Ärzten für Sie erstellt worden sind. Sie führen Sie durch alle wichtigen Themen Ihres Fachgebiets und unterstützen Sie bei der Vorbereitung auf die Facharztprüfung. Basierend auf der Grundlage der Muster-Weiterbildungsordnung der Ärztekammer wurden die Weiterbildungscurricula entwickelt und genau auf die Bedürfnisse angehender Fachärztinnen und Fachärzte zugeschnitten. „Gerade im klinischen Alltag und in den verschiedenen Schwerpunktbereichen, die man bis zur Facharztprüfung durchläuft, fällt es manchmal schwer, praxisnahe und relevante Inhalte für die unterschiedlichen Anforderungsfälle zu finden “, berichten die Herausgeberinnen und die Herausgeber eines der Weiterbildungscurricula. Hierbei sind die gut strukturierten „Leselisten“ eine große Hilfe, denn die eRef bietet digitalen Zugriff auf die Inhalte der medizinischen Fachbücher und Zeitschriften von Thieme, ein umfangreiches Bildarchiv, Videos, und viele weitere Services - für alle Fachgebiete der Medizin. All diese Informationen sind inner- halb der Weiterbildungscurricula fachspezifisch für Sie gebündelt und für das schnelle Nachschlagen, die strukturierte Vorbereitung auf die Facharztprüfung oder als Refresher für die nächste Rotation konzipiert. Zudem lässt sich der persönliche Lernfortschritt vermerken und zur Wissenskontrolle stehen Prüfungsfragen und Fallbeispiele im Trainingscenter zur Verfügung. Wenn Sie gern Ihre eigene Playlist zusammenstellen möchten, ist das natürlich auch möglich: losgelöst vom Weiterbildungscurriculum können im persönlichen eRef Account eigene Playlisten anlegt werden, ganz individuell zu den Themen und mit den Inhalten, die Sie benötigen.

Schauen Sie in das Weiterbildungscurriculum für Ihr Fach doch direkt mal rein:

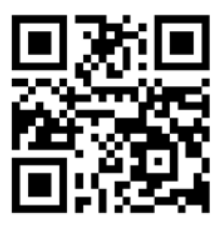

https://eref-testen.thieme.de/produkt/ weiterbildungscurricula/ 